\title{
Les améloblastomes : étude rétrospective
}

\section{Ameloblastoma : a retrospective study}

\section{MOTS-CLEFS :}

- Améloblastome, tumeurs maxillaires, chirurgie conservatrice, histologie, récidive

KEYWORDS:

- Ameloblastoma maxillary tumors, conservative surgery, histology, recurrence

$\operatorname{AOS} n^{\circ} 284-2017$

\section{Résumé}

Introduction : L'améloblastome est une tumeur bénigne relativement rare. C'est un néoplasme vrai de l'organe de l'émail. Cette tumeur est caractérisée par sa remarquable latence mais aussi par son agressivité et sa tendance ò la récidive.

Matériels et méthode : il s'agit d'une étude rétrospective sur 11 ans (2002-2012) portant sur 22 patients. Les données recueillies ont été traitées et analysées par le logiciel Excel 2007.

Résultats : cette tumeur touche l'adulte jeune avec prédominance masculine. $95 \%$ des patients consultent pour une tuméfaction mandibulaire ( $100 \%)$ la plupart du temps unique $(90 \%)$ et postérieure (76,2\%). L'examen radiologique standard (panoramique) systématique a montré une image polygéodique dans $67 \%$ des cas. Le traitement de cette tumeur était chirurgical pour tous les patients avec ou sans réparation.

Conclusion : bien que la prise en charge thérapeutique de la majorité des tumeurs améloblastiques soit du ressort du chirurgien maxillo-facial en Tunisie, le rôle du médecin dentiste reste néanmoins important : d'une part pour établir un diagnostic précoce surtout que cette tumeur engendre des signes dentaires variés et également dans la prise en charge prothétique post-chirurgicale.
Abstract

Introduction: Ameloblastoma is a relatively rare benign tumor. This is a true neoplasm of the enamel organ. This tumor is characterized by its remarkable latency and also its aggressiveness and high recurrence rate.

Materials and methods: This is a retrospective study over 11 years (2002-2012) on 22 patients. The data were processed and analyzed by the Excel 2007 software.

Results: This tumor affects young adults with male predominance. 95\% of patients presented most frequently with a single (90\%) and posterior (76.2\%) mandibular swelling. The standard radiological exam (panoramic radiography) showed a multilocular radiolucency in $67 \%$ of cases. The treatment was surgery for all patients with or without immediate repair.

Conclusion: Although the management of the majority of ameloblastic tumors is the privilege of the maxillofacial surgeon in Tunisia, the role of the dentist is paramount: an early diagnosis taking into account the several dental signs and also a prosthetic rehabilitation.

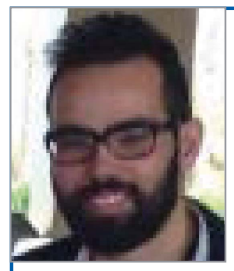

- Marouene BEL HADJ HASSINE, Résident en Médecine et Chirurgie buccales, DDS, Département de Médecine Dentaire, CHU Sahloul, Sousse, Tunisie. Marouene_belhadjhassine@hotmail.fr

Lamia OUALHA, Professeur en Médecine et Chirurgie Buccales, DDS, Département de Médecine Dentaire, CHU Sahloul, Sousse, Tunisie.

Samia AYACHI, Professeur Agrégé en Chirurgie Maxillo-Faciale, MD, Département de Chirurgie Maxillo-Faciale, CHU Sahloul, Sousse, Tunisie.

S. Kalaï, Résidente en Médecine et Chirurgie Buccales, DDS, Département de Médecine Dentaire, CHU Sahloul, Sousse, Tunisie.

H. KHOCHTALI, Professeur et chef du Service de Chirurgie Maxillo-Faciale, MD, Département de Chirurgie Maxillo-Faciale, CHU Sahloul, Sousse, Tunisie.

Nabiha DOUKI, Professeur en Odontologie Conservatrice et Endodontie et chef du Service de Médecine Dentaire, DDS, Département de Médecine Dentaire, CHU Sahloul, Sousse, Tunisie. 


\section{INTRODUCTION}

L'améloblastome est une tumeur bénigne odontogénique des maxillaires, localement agressive, dont l'origine est l'épithélium impliqué dans la formation des dents à savoir l'organe de l'émail, les restes épithéliaux de Malassez, la paroi de kystes odontogéniques (dentigères) ou également les cellules épithéliales basales de la muqueuse orale. L'OMS a fait la distinction entre les améloblastomes bénins et les améloblastomes malins ${ }^{1}$. Dans le premier groupe, 4 types ont été identifiés : améloblastome solide ou multykystique, améloblastome desmoplastique, améloblastome unikystique et améloblastome extraosseux ou périphérique. Les améloblastomes malins sont extrêmement rares et regroupent les améloblastomes métastatiques et les carcinomes améloblastiques ${ }^{2}$.

Son étiopathogénie est toujours mal élucidée bien que quelques thèses étiologiques aient été avancées concernant les extractions, les caries, les traumatismes, l'inflammation et les déficits nutritionnels ${ }^{3}$.

Cliniquement, les améloblastomes peuvent rester asymptomatiques avant la découverte d'une tuméfaction faciale. En revanche, sur le plan radiologique, ils se présentent sous la forme d'une lésion ostéolytique radiotransparente uni ou multiloculaire d'allure kystique. Parfois, des septums osseux intralésionnels sont visibles donnant un aspect en nid d'abeille. L'expansion corticale, aussi bien vestibulaire que linguale, est plus fréquente par rapport aux tumeurs kératokystiques odontogéniques et la résorption radiculaire est un signe important mais inconstant. Le diagnostic différentiel se pose avec les kystes odontogéniques, les tumeurs kératokystiques odontogéniques, les myxomes et également avec des tumeurs non odontogéniques tels que les granulomes centraux à cellules géantes et les kystes solitaires ${ }^{2}$.

Le but de cet article est de déterminer les aspects épidémiologiques des améloblastomes au sein d'une population tunisienne, de préciser les caractéristiques radio-cliniques de ces lésions, leurs aspects thérapeutiques en les comparant à ceux de la littérature. Enfin, on essayera de mettre l'accent sur le rôle du médecin dentiste dans la prise en charge de tels patients.

\section{MATÉRIELS ET MÉTHODE}

C'est une étude rétrospective étalée sur 11 ans entre janvier 2002 et décembre 2012, portant sur 22 patients colligés au service de chirurgie maxillo-facial du C.H.U Sahloul (Sousse Tunisie).

Les critères d'inclusion: l'étude a porté sur tout patient, homme ou femme, opéré pour un améloblas- tome suite à un examen clinique et radiographique évoquant un tel diagnostic.

Données recueillies : l'ensemble des dossiers et fiches ont été relus et les données recueillis ont été notées sur un formulaire d'exploitation conçue aux fins de la présente étude.

Ces données concernent les informations administratives : le numéro d'entrée, âge, sexe et le lieu de résidence.

I Le motif et le délai de consultation ont été notés.

- Les antécédents généraux et locaux.

- L'examen clinique exobuccal et endobuccal avec des données de l'inspection et de la palpation.

I Les résultats des examens para cliniques ont été notés.

- Le traitement effectué a été mentionné par le type de l'intervention, l'association ou non à une radio et ou chimiothérapie et l'association ou non à une réparation chirurgicale ainsi que son type.

$\checkmark$ La réponse définitive de l'examen anatomopathologique qui a déterminé le type histologique de la tumeur améloblastique.

\ Le suivi et l'évolution ont pu être notés sur quelques dossiers alors que beaucoup de patients ont été perdus de vue.

Ces données ont ensuite été traitées et analysées par le logiciel Microsoft Office Excel 2007.

\section{RÉSULTATS}

L'âge moyen des patients était de 36 ans et demi, avec des extrêmes allant de 3 ans à 75 ans. La tranche d'âge la plus touchée est celle de $30-40$ ans $(36,36 \%)$. Une prédominance masculine a été notée (59\%).

Pour le délai de consultation $30 \%$ des patients consultaient en moins de 4 mois et $30 \%$ entre 1 et 5 ans.

Presque la totalité des patients (95\%) ont consulté pour une tuméfaction mandibulaire engendrant soit une asymétrie faciale soit une gêne fonctionnelle, alors que la douleur n'est rapportée que par un tiers des patients. Les signes dentaires ont varié d'une algie à la chute spontanée.

Tous les améloblastomes étaient mandibulaires, avec $76,2 \%$ des cas à localisation postérieure ; $90 \%$ des cas présentaient une localisation unique et le côté droit était le plus touché soit $65 \%$ des cas étudiés.

Le tiers des patients a mentionné une hypoesthésie $\mathrm{du} \mathrm{V}_{3}$; et seulement deux patients se sont présentés avec un trismus.

L'inspection a révélé que la peau de recouvrement n'était ulcérée ou enflammée que dans $10 \%$ des cas. La palpation, douloureuse chez $55,5 \%$ des patients, a montré une tuméfaction volumineuse $(>4 \mathrm{~cm})$ dans 
$61 \%$ des cas, elle était de consistance dure chez $81 \%$ des cas ; les limites de la tuméfaction n'ont pas été précisées dans tous les dossiers. Les adénopathies n'ont été retrouvées que chez deux patients.

L'examen endobuccal a précisé que la muqueuse était saine pour $76 \%$ des patients, sinon elle était soit érythémateuse soit présentait une fistule. Les signes dentaires les plus fréquents étaient la douleur dentaire $(38,09 \%)$ et les déplacements dentaires $(38,09 \%)$.

Les examens complémentaires comportaient un ortho-pantomogramme pour 21 patients sur 22 ; cet examen a montré que le côté droit de la mandibule était le plus touché (70\%) avec une localisation préférentielle au niveau de la région prémolo-molaire. L'image était polygéodique pour les deux tiers des cas mais toujours homogène et à contours réguliers. Le canal mandibulaire paraissait refoulé dans $52 \%$ des cas. Vingt patients ont bénéficié d'un examen tomodensitométrique, $21 \%$ des cas présentaient une image inhomogène avec pour $68,18 \%$ des cas les corticales étaient rompues. Les signes de malignité ont été retrouvés chez six de nos patients à savoir l'envahissement du canal mandibulaire ainsi que l'aspect en dentelle traduisant l'envahissement des parties molles malgré l'absence de signes cliniques en faveur de la malignité.

Le traitement était exclusivement chirurgical pour tous les patients sans radio ni chimiothérapie. L'énucléation a été pratiquée chez $32 \%$ des cas alors que le traitement radical comportait la résection partielle et totale pour 12 patients $(54 \%)$ et l'hémimandibulectomie pour 3 patients ( $14 \%)$.

Les procédés de réparation chirurgicale employés étaient l'autogreffe iliaque (5 patients) ou costale (2 patients) ou bien par attelles métalliques ou ostéosynthèse par fils en acier.

L'examen anatomopathologique systématique a montré cinq types histologiques des améloblastomes: $54,5 \%$ de type folliculaire ; $18,2 \%$ de type plexiforme ; $9,1 \%$ de type granuleux ; $4,5 \%$ de type acanthomateux ou desmoplasique et aussi deux fibromes améloblastiques.

\section{DISCUSSION}

L'améloblastome est une tumeur relativement rare en Tunisie, puisque seuls 22 cas ont été recensés sur une période de 11 ans, soit une moyenne de 2 cas par an ; incidence proche de celle rapportée dans d'autres pays africains (Tableau 1).

L'âge moyen, selon notre série est 36 ans et demi, ce qui est en concordance avec la majorité des études prouvant que l'améloblastome constitue une tumeur bénigne de l'adulte jeune (Tableau 2).

Une légère prédominance masculine a été relevée avec un sexe ratio de 1.44/1. En effet, dans une revue systématique de Seintou et al., il s'avère qu'il n'y a pas d'évidence laissant suggérer qu'un sexe soit plus susceptible aux améloblastomes que l'autre. En revanche, d'autres études ont montré une plus forte incidence

\begin{tabular}{|c|c|c|c|}
\hline Auteurs & Année & Pays & Incidence \\
\hline Nastri Al et coll. & 1995 & Australie & $<1$ cas par an \\
\hline Junquera L et coll. & 2003 & Espagne & $<1$ cas par an \\
\hline Christos CG et coll. & 2000 & Ethiopie & 3 cas par an \\
\hline Crezoit GE et coll. & 2003 & Côte d'Ivoire & 3 cas par an \\
\hline Olaïtan AA et coll. & 1993 & Nigéria & 16 cas par an \\
\hline Ruhin-Poncet et coll. & 2011 & France & 18,38 par an \\
\hline
\end{tabular}

$\triangle$ Tableau 1 : Incidence des améloblastomes selon différentes études réalisées dans le monde

\begin{tabular}{|c|c|c|c|}
\hline Auteurs & Année & Pays & Age moyen \\
\hline Jebloui Y et coll. & 2007 & Tunisie & 35 ans \\
\hline Diakhere SMG et coll. & 2007 & Sénégal & 37 ans \\
\hline Saoud S et coll. & 2010 & Maroc & 38 ans \\
\hline Bou-el-Harmel FZ et coll. & 2008 & Maroc & 39 ans \\
\hline Kim SG et coll. & 2001 & Corée du nord & 30,4 ans \\
\hline Siar CH et coll. & 1993 & Malaisie & 30,8 ans \\
\hline Vignikin-yehouessi B et coll. & 2007 & Bénin & 31,8 ans \\
\hline Ruhin-Poncet et coll. & 2011 & France & 36,4 ans \\
\hline
\end{tabular}

$\triangle$ Tableau 2 : Âge moyen de survenu de l'améloblastome selon différentes études réalisées dans le monde 
chez les femmes ${ }^{4,5}$. Cliniquement, la tuméfaction faciale constitue le motif de consultation le plus fréquent à travers différentes études menées ${ }^{6,7,8}$ (Fig. 1a, b, c). Cette tuméfaction est le plus souvent indolore, alors que le tiers de nos patients ont rapporté des douleurs contre 7, $5 \%$ pour Jeblaoui $\mathrm{Y}$ et al. et $18 \%$ pour Ruhin-Poncet et $\mathrm{al}^{9}$.

Devant le silence clinique de l'améloblastome, le délai de consultation semble relativement long surtout dans les pays africains et ceci peut s'expliquer par l'absence ou la difficulté d'accès aux soins et aux structures hospitalières spécialisées. En effet, les patients ne consultent qu'après l'installation de signes invalidants ce qui est probablement la raison de la fréquence des améloblastomes dont la taille est importante. D'ailleurs, 76, $47 \%$ des patients avaient des lésions de plus de $4 \mathrm{~cm}$. En revanche, dans les pays développés, les améloblastomes sont fréquemment détectés au cours des visites de contrôle comme le démontre l'étude de Buchner et $\mathrm{al}^{10}$.
Le deuxième motif de consultation n'est autre que la découverte fortuite d'une lésion ostéolytique lors des RDV de contrôle chez l'orthodontiste suivi par la sensation d'une légère pesanteur au niveau de la joue et/ou l'apparition de douleurs associées.

Le panoramique dentaire constitue l'examen radiologique de première intention, permettant souvent d'orienter le diagnostic : $67 \%$ des sujets examinés ont présenté un aspect polygéodique ce qui est conforme avec plusieurs études. Toutefois, une lecture tridimensionnelle de la lésion est nécessaire pour en préciser la nature, le contour, l'état des corticales, la situation par rapport aux structures anatomiques et la présence ou non de micro géodes en périphérie de l'image principale ou au niveau des cloisons intergéodiques (Fig. 2). La prise de contraste trouvée dans $14 \%$ des cas est expliquée par la présence de composantes charnues et de parois kystiques qui se rehaussent après injection du produit de contraste comme l'a précisé Saoud S. La mobilité dentaire est dûe à l'amincissement

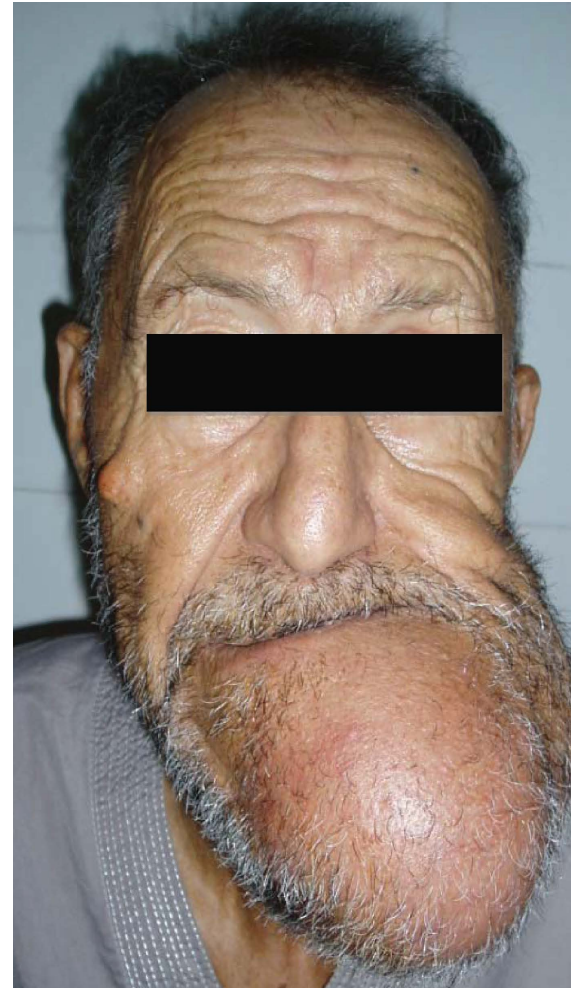

$\triangle$ Fig. 1a: Vue de face d'un patient présentant une importante tuméfaction mandibulaire gauche.

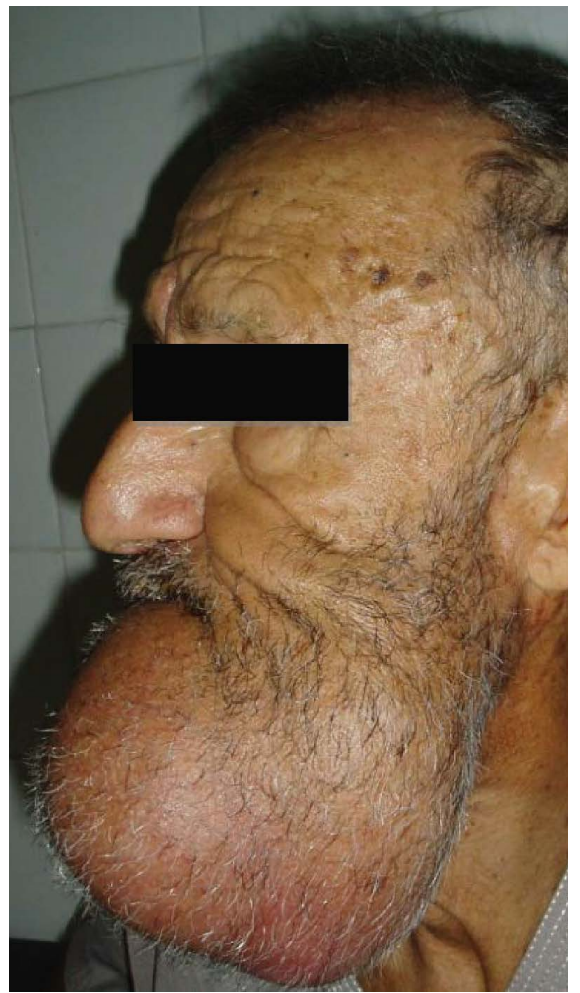

$\triangle$ Fig. lb: Vue de profil.

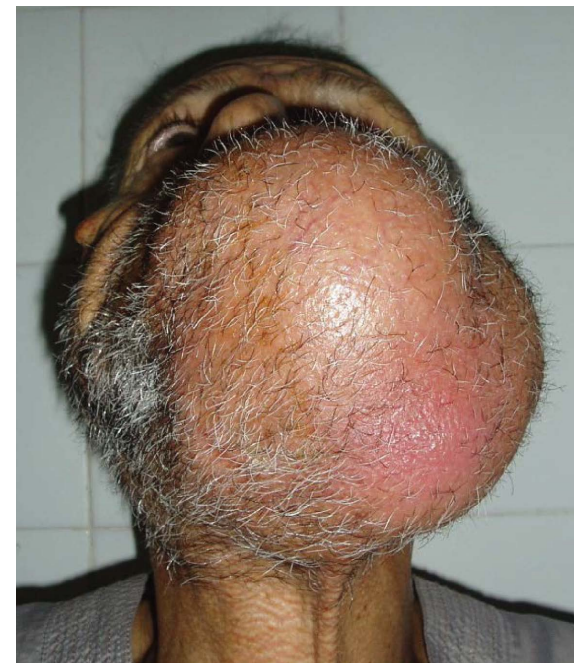

$\triangle$ Fig. 1c: Vue inférieure.

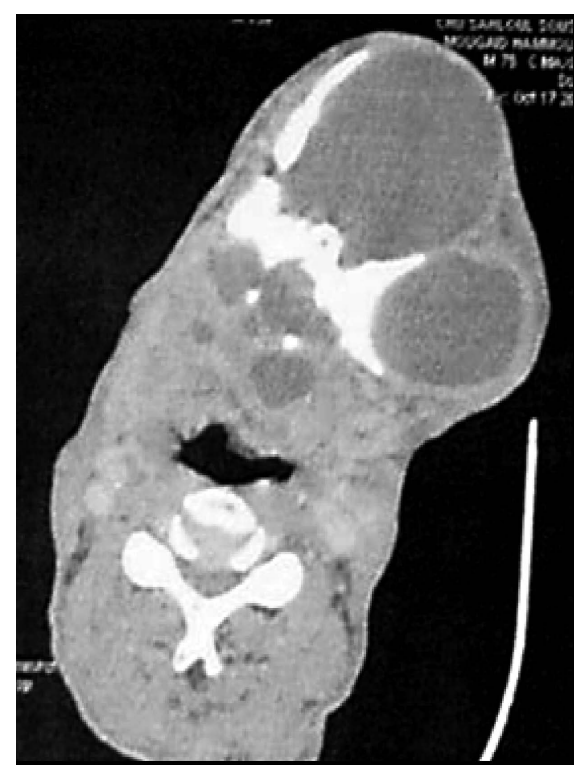


des corticales, quant aux troubles sensitifs, notamment l'hypoesthésie qui est rapportée par le tiers de nos patients, elle serait liée au refoulement du nerf alvéolaire inférieur.

Après un examen clinique et radiologique minutieux le médecin dentiste devrait être capable de poser le diagnostic d'une tumeur bénigne et en cas de doute une collaboration avec un spécialiste est nécessaire.

Le diagnostic final est confirmé par l'examen histologique: pour l'améloblastome multikystique, on trouve 3 sous-types histologiques : folliculaire, plexiforme et granulaire. Notre étude a permis de démontrer que le type folliculaire est de loin le plus fréquent avec $54,5 \%$ des cas, suivi par le type plexiforme avec $18,2 \%$. Plusieurs études ont supposé que les types granulaire et desmoplastique seraient les plus agressifs, ce qui n'est plus vrai de nos jours ${ }^{11}$. En effet, l'améloblastome solide ou multikystique, indépendamment de la nature histologique, doit être considéré comme étant une tumeur bénigne mais agressive avec un fort potentiel de récidive. L'étude de coupes histologiques provenant de mandibule réséquée a montré que les cellules améloblastiques peuvent pénétrer l'os jusqu'à $8 \mathrm{~mm}$ au-delà des limites cliniques et radiologiques de la lésion. Par conséquent, le traitement conservateur, en l'occurrence l'énucléation, serait responsable d'un taux de récidive important avoisinant les $60-80 \%{ }^{12,13,14,15}$. D'où le recours à la résection large avec $1 \mathrm{~cm}$ de marges autour de la lésion. Le nerf alvéolaire inférieur est souvent sacrifié malgré la possibilité de reconstruction moyennant une greffe de nerf si besoin, mais en général, la majorité des patients le tolère sans difficulté. D’autres techniques de résection mandibulaire préservatrice du nerf alvéolaire inférieur ont été décrites, cependant, le risque de récidive demeure important à cause de la présence de cellules améloblastiques résiduelles adhérentes au nerf.

$\mathrm{Au}$ maxillaire, les lésions sont plus difficiles à gérer bien qu'elles soient identiques de point de vue histologique. En effet, les tumeurs postérieures maxillaires ont plus de facilité d'infiltrer l'espace ptérygomaxillaire voire même la base du crâne, d'où la nécessité de recourir à une maxillectomie partielle ou totale ${ }^{16,17}$. La reconstruction de la perte de substance mandibulaire se fait généralement aux moyens d'une plaque en titanium en première phase, s'en suit une greffe osseuse associée à une greffe de peau et la mise en place d'implants ${ }^{18,19,12,20,21,22}$. Une autre option thérapeutique s'offre au chirurgien maxillo-facial à savoir le recours aux lambeaux libres pour la reconstruction aussi bien des tissus durs et mous.

$\mathrm{Au}$ regard des séquelles de la résection osseuse et compte tenu de l'âge du patient, essentiellement les plus jeunes d'entre eux, d'autres alternatives ont été proposées comme l'énucléation avec ou sans curetage complétée par une technique permettant l'élimination des cellules améloblastiques résiduelles. Ces techniques incluent l'utilisation du nitrogène liquide ou encore la solution de Carnoy. Les résultats sont plutôt probants étant donné le taux de récidive qui est de l'ordre de $16 \%$ par rapport à la résection, de loin le traitement de choix avec $5,9 \%$ de récidive ${ }^{22}$.

Concernant les améloblastomes unikystiques, le premier cas a été décrit en 1977 par Robinson et Marti$n e z^{23}$, il touche plus fréquemment les sujets jeunes, hommes et femmes d'une manière quasi égale, avec plus de $50 \%$ des cas au cours de la deuxième décade. Le corps de la mandibule est le site de prédilection avec un aspect radiologique uniloculaire dans la majorité des cas. Le traitement reste tout de même controversé : un traitement conservateur est, tout de même, préconisé malgré un taux de récidive élevé, et ce dans le but d'assurer une meilleure qualité de vie pour les patients, essentiellement les plus jeunes.

L'analyse de Seintou et al, a pu mettre en évidence que le sous-type luminal est le moins agressif, d'où un meilleur pronostic, en revanche les variantes plexiforme et murale sont souvent associées à un taux de récidive important. Par conséquent, d'autres études sont nécessaires afin d'établir un plan de traitement optimal.

L'améloblastome périphérique est une tumeur qui touche les tissus mous sans envahissement de l'os. Cliniquement, il se présente sous la forme d'une masse mandibulaire dans $70 \%$ des cas, indolore, sessile, ferme, d'aspect verruqueux. D'un point de vue histologique, il est identique aux autres types d'améloblastomeintraosseux, prenant origine de l'épithélium de surface ou les restes extra osseux de la lame dentaire. Le traitement conservateur est souvent indiqué avec un risque de récidive quasi nul.

La phase postchirurgicale est d'une importance capitale car, souvent elle permet de suivre l'évolution et de dépister à temps les récidives qui restent tout de même fréquentes. Sammartino et $\mathrm{al}^{25}$, ont suggéré un contrôle radiologique tous les 6 mois pendant les 5 premières années et un contrôle annuel pendant les 5 années suivantes; une TDM à 5 ans post opératoire ou en cas de récidive décelée sur le panoramique dentaire.

En outre, plusieurs auteurs ont rapporté des récidives après 25 ans $^{26,27}$, d'où un contrôle radiologique tous les 2-3 ans une fois les 10 ans de suivi sont dépassés. À côté du traitement de l'améloblastome, la réhabilitation prothétique, post reconstruction mandibulaire, revêt une importance capitale non seulement dans un but esthétique mais surtout fonctionnel. 
Toutefois l'objectif est rarement obtenu du fait de l'état dentaire préexistant, la qualité de l'hygiène bucco-dentaire, le niveau socio-économique des patients traités dans le service. En effet, il n'est pas rare que des prothèses soit confectionnées mais inutilisées par le patient.
Le médecin dentiste, outre son rôle dans la prise en charge prothétique, est impliqué dans le diagnostic de l'améloblastome compte tenu de son expression clinique ainsi que dans le dépistage des récidives. D’où, la nécessité de motiver les patients à se présenter aux RDV de contrôle.

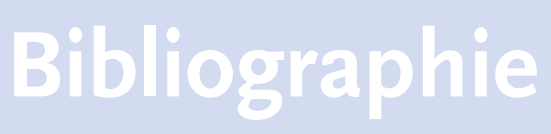

[1] Barnes L, Eveson JW, Reichart PA, Sidransky D. World Health Organization Classification of Tumours. Pathology and Genetics. Head and Neck Tumours. Lyon : World Health Organization International Agency for Research on Cancer, IACR Press ; 2005.

[2] Hertog D, van der Waal I. Ameloblastoma of the jaws : a critical reappraisal based on a 40 years single institution experience. Oral Oncol. 2010 Jan ; 46(1):61-4.

[3] Kim SG, Jang HS. Ameloblastoma : a clinical, radiographic, and histopathologic analysis of 71 cases. Oral Surg Oral Med Oral Pathol Oral Radiol Endod. 2001 Jun ; 91(6):649-53.

[4] Olaitan AA1, Adekeye EO. Clinical features and management of ameloblastoma of the mandible in children and adolescents. $\mathrm{Br} \mathrm{J}$ Oral Maxillofac Surg. 1996 Jun ; 34(3):248-51.

[5] Daramola JO, Ajagbe HA, Oluwasanmi JO. Ameloblastoma of the jaws in Nigerian children. A review of sixteen cases. OralSurg Oral Med Oral Pathol. 1975 Oct ; 40(4):458-63.

[6] Zhang J, Gu Z, Jiang L, Zhao J, Tian M, Zhou J, Duan Y. Ameloblastoma in children and adolescents. Br J Oral Maxillofac Surg. 2010 Oct ; 48(7):549-54.

[7] Ledesma C, Garces M, Ibarra A, Portilla J, Hernandez JC. Ameloblastoma in Latin America. Analysis of 338 cases. Med Oral. 2000 Aug-Oct ; 5(4):254-260.

[8] Arotiba GT, Ladeinde AL, Arotiba JT, Ajike SO, Ugboko VI, Ajayi OF. Ameloblastoma in Nigerian children and adolescents : a review of 79 cases. J Oral Maxillofac Surg. 2005 Jun ; 63(6):747-51.
[9] Ruhin-Poncet B, Bouattour A, Picard A, Menard P, Capron F, Bertrand J-C. Améloblastomes des machoires. Analyse rétrospective de 1994 à 2007. Rev Stomatol Chir Maxillofac 2011 ; 112:269-279.

[10] Buchner A, Merrell PW, Carpenter WM. Relative frequency of central odontogenic tumors : a study of 1,088 cases from Northern California and comparison to studies from other parts of the world. J Oral Maxillofac Surg. 2006 Sep ; 64(9):1343-52.

[11] Ueno S, Mushimoto K, Shirasu R. Prognostic evaluation of ameloblastoma based on histologic and radiographic typing. J OralMaxillofacSurg. 1989 Jan ; 47(1):11-5.

[12] Ghandhi D, Ayoub AF, Pogrel MA, MacDonald G, Brocklebank LM, Moos KF. Ameloblastoma : a surgeon's dilemma. J Oral MaxillofacSurg. 2006 Jul ; 64(7):1010-4.

[13] Mehlisch DR, Dahlin DC, Masson JK. Ameloblastoma : a clinicopathologic report. J OralSurg. 1972 Jan ; 30(1):9-22.

[14] Shatkin S, Hoffmeister FS. Ameloblastoma : a rational approach to therapy. Oral Surg Oral Med Oral Pathol. 1965 Oct ; 20(4):421-35.

[15] Waldron CA. Ameloblastoma in perspective. J OralSurg. $1966 \mathrm{Jul}$; 24(4):331-3.

[16] Nastri AL, Wiesenfeld D, Radden BG, Eveson J, Scully C. Maxillary ameloblastoma : a retrospective study of 13 cases. $\mathrm{Br} J$ Oral Maxillofac Surg. 1995 Feb ; 33(1):28-32.

[17] Zwahlen RA, Grätz KW. Maxillary ameloblastomas : a review of the literature and of a 15-year database. J Craniomaxillofac Surg. 2002 Oct ; 30(5):273-9.

[18] Bataineh AB. Effect of preservation of the inferior and posterior borders on recurrence of ameloblastomas of the mandible. Oral Surg Oral Med Oral Pathol Oral Radiol Endod. 2000 Aug ; 90(2):155-63.
[19] Carlson ER, Marx RE. The ameloblastoma : primary, curative surgical management. J Oral Maxillofac Surg. 2006 Mar ; 64(3):484-94.

[20] Junquera L, Ascani G, Vicente JC, García-Consuegra L, Roig P. Ameloblastoma revisited. Ann Otol Rhinol Laryngol. 2003 Dec; 112(12):1034-9.

[21] Nakamura N, Higuchi Y, Mitsuyasu T, Sandra F, Ohishi M. Comparison of longterm results between different approaches to ameloblastoma. Oral Surg Oral Med Oral Pathol Oral RadiolEndod. 2002 Jan ; 93(1):13-20.

[22] Sachs SA. Surgical excision with peripheral ostectomy : A definitive, yet conservative, approach to the surgical management of ameloblastoma. J Oral Maxillofac Surg. 2006 Mar ; 64(3):476-83.

[23] Lee PK, Samman N, Ng IO. Unicystic ameloblastoma--use of Carnoy's solution after enucleation. Int J Oral Maxillofac Surg. 2004 Apr ; 33(3):263-7.

[24] Robinson L, Martinez MG. Unicystic ameloblastoma : a prognosticallydistinctentity. Cancer. 1977 Nov ; 40(5):2278-85.

[25] Sammartino G, Zarrelli C, Urciuolo V, di Lauro AE, di Lauro F, Santarelli A, Giannone N, Lo Muzio L.Effectiveness of a new decisional algorithm in managing mandibular ameloblastomas : a 10-years experience. $\mathrm{Br} \mathrm{J}$ Oral Maxillofac Surg. 2007 Jun ; 45(4):306-10. Epub 2006 Oct 23.

[26] Demeulemeester LJ, Mommaerts MY, Fossion E, Bossuyt M. Late locoregional recurrences after radical resection for mandibular ameloblastoma. Int J Oral Maxillofac Surg. 1988 Oct ; 17(5):310-5.

[27] Zachariades N. Recurrences of ameloblastoma in bone grafts. Report of 4 cases. Int J Oral Maxillofac Surg. 1988 Oct;17(5):316-8. 